\title{
Author Correction: Graphene bilayers with a twist
}

Eva Y. Andrei ${ }^{D}$ and Allan H. MacDonald

Correction to: Nature Materials https://doi.org/10.1038/s41563-020-00840-0, published online 18 November 2020.

In the version of this Review Article originally published, in Fig. 1e the redundant labels $\Gamma_{s}, K_{s}^{\prime}, M_{s}$ and $K_{s}$ mistakenly appeared in the upper black hexagon; these labels have been removed in the online versions of the Review Article.

Published online: 5 January 2021

https://doi.org/10.1038/s41563-020-00917-w

() Springer Nature Limited 2021

\section{Author Correction: A general Lewis acidic etching route for preparing MXenes with enhanced electrochemical performance in non-aqueous electrolyte}

Youbing Li, Hui Shao, Zifeng Lin DD, Jun Lu, Liyuan Liu, Benjamin Duployer, Per O. Å. Persson, Per Eklund (D,

Lars Hultman, Mian Li, Ke Chen, Xian-Hu Zha, Shiyu Du, Patrick Rozier ID, Zhifang Chai, Encarnacion Raymundo-Piñero,

Pierre-Louis Taberna (D), Patrice Simon (D) and Qing Huang (D)

Correction to: Nature Materials https://doi.org/10.1038/s41563-020-0657-0, published online 13 April 2020.

In the Supplementary Information file originally published for this Article, Supplementary Fig. 21 was mistakenly a duplicate of Supplementary Fig. 17. The Supplementary Information file has now been updated with the correct version of Supplementary Fig. 21.

Published online: 18 January 2021

https://doi.org/10.1038/s41563-021-00925-4

(c) The Author(s), under exclusive licence to Springer Nature Limited 2021 